\title{
Novel endoscopic treatment for colocutaneous fistula after severe acute pancreatitis: filling with a polyglycolic acid sheet
}

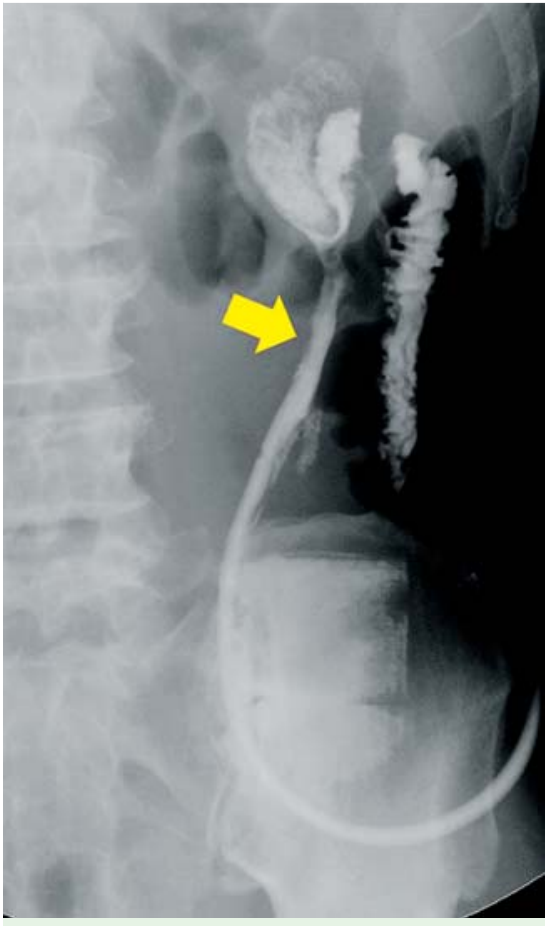

Fig. 1 Contrast imaging performed by introducing a contrast medium through the abdominal drain revealed a colocutaneous fistula (arrow).

The formation of gastrointestinal fistulas has been reported as a rare complication associated with late-stage, severe, acute pancreatitis. This condition is difficult to treat and often requires surgery [1]. Here we report on a successful case of endoscopically implanted polyglycolic acid (PGA) sheets (Neoveil; Gunze Co., Tokyo, Japan) being used to repair a fistula that had formed between the colon and skin.

A 60-year-old man had been hospitalized for alcohol-related severe acute pancreatitis. Because he suffered from extensive walled-off necrosis, we performed necrosectomy and colostomy with double orifices in the transverse colon. Owing to the complications of acute respiratory distress syndrome and acute renal failure, the patient was observed for 2 months in the intensive care unit, after which he was transferred to the general ward. Contrast imaging performed by introducing a contrast medium through the abdominal drain revealed a colocutaneous fistula ( $\bullet$ Fig.1). Endoscopic examination indi-

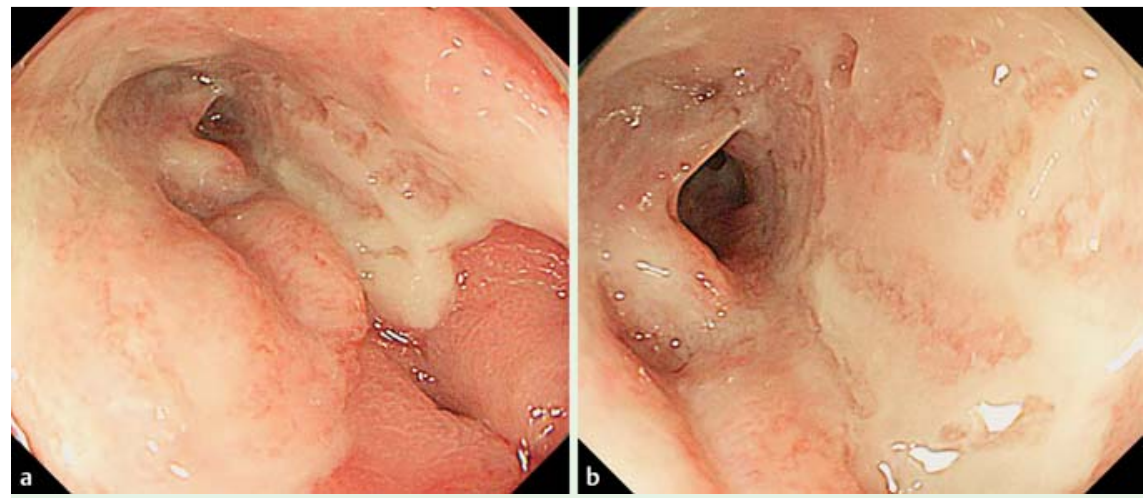

Fig.2 Endoscopic images. a Endoscopy confirmed stenosis and ulceration of the splenic flexure. b The fistula was in the center of the ulcer.

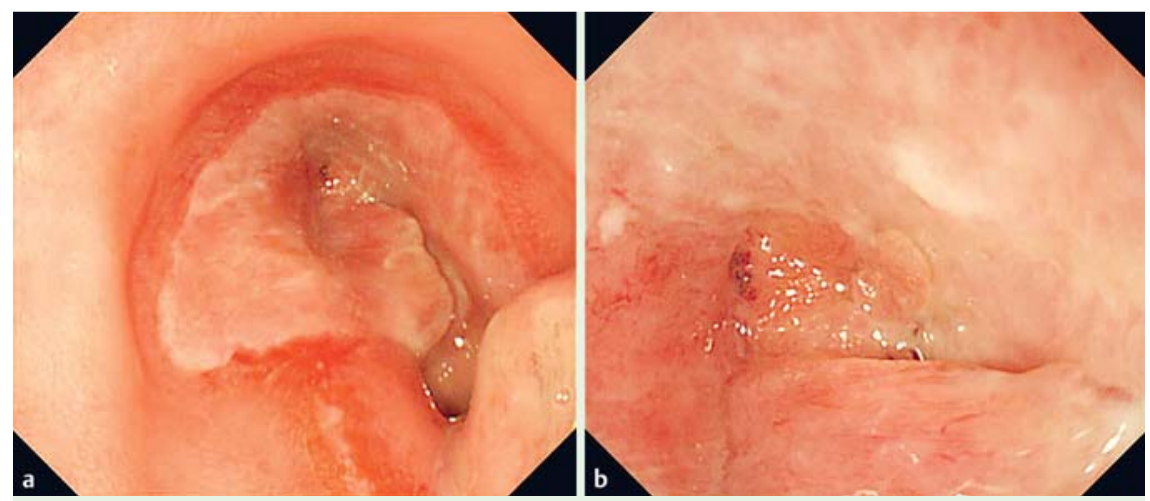

Fig. 3 Endoscopic images 3 weeks after polyglycolic acid sheet application. a, b The fistula was covered by granulation tissue and completely closed.

cated ulceration and stenosis of the splenic flexure along with a fistula ( $\bullet$ Fig. 2 ). Follow-up examination was performed a month later, and results indicated that the fistula had not closed. We then decided to perform endoscopic insertion of PGA sheets into the fistula.

An endoscope was inserted through the colostomy and maneuvered to the fistula. The forceps grasping the PGA sheet was inserted through the endoscope. The PGA sheet extending from the end of the endoscope was grasped by another forceps that was inserted through the fistula and then pulled up toward the skin. This procedure was repeated three times to fill the fistula with several PGA sheets. An endoscopic sprinkler was then used to apply a fibrin sealant (Beriplast P Combi-Set Tissue adhesion; CSL Behring, Tokyo, Japan) and complete the procedure ( $\bullet$ Video 1 ).

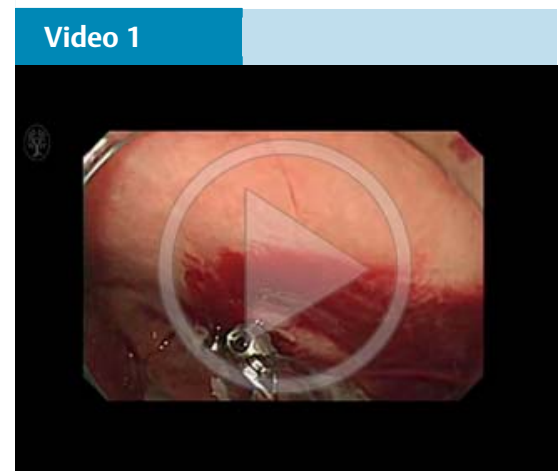

Endoscopic insertion of polyglycolic acid sheets into the fistula.

Endoscopic images taken 3 weeks after the procedure showed that the fistula had been covered by granulation tissue and had completely closed ( Fig. 3). The 


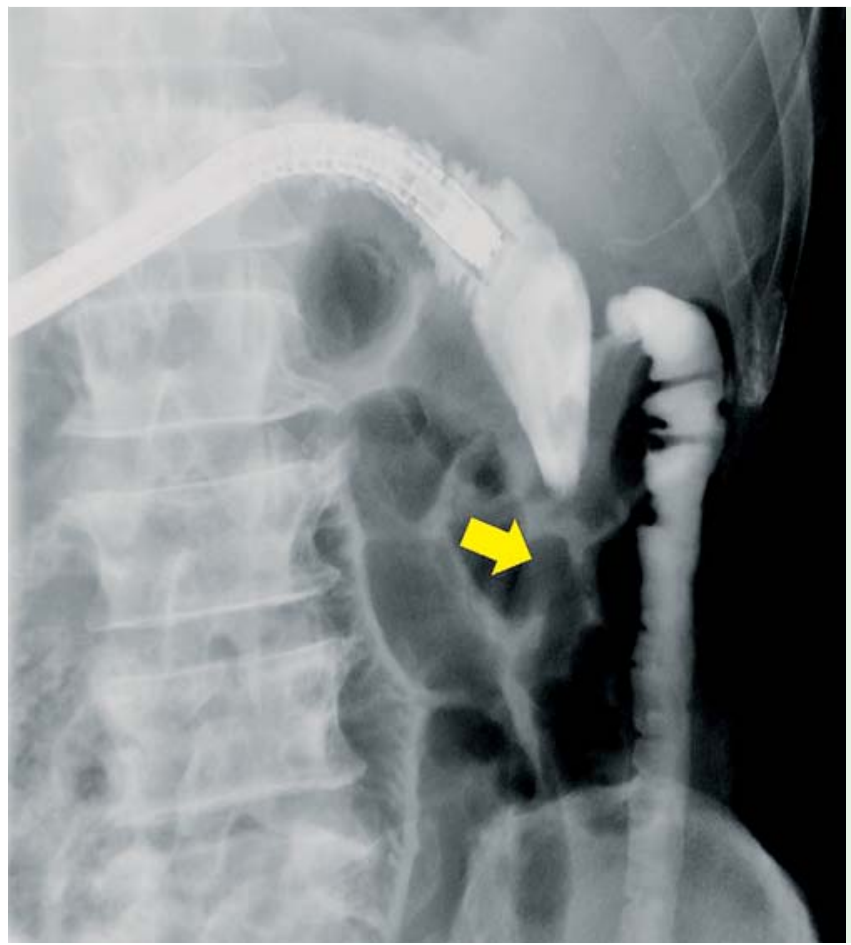

Fig. 4 The fistula on contrast radiograms, even after pressure injection of the contrast medium.

fistula was not detectable on contrast radiograms, even after pressure injection of the contrast medium ( $\bullet$ Fig. 4 ).

PGA promotes the formation of granulation tissue and is a reinforcing material that is naturally absorbed by the body ( $\bullet$ Fig. 5). In recent years, PGA has been gaining attention in the field of gastrointestinal endoscopy. It is being used to prevent stenosis and perforations, and to close fistulas after endoscopic submucosal dissection $[2,3]$. The outcome in the present case indicates that the endoscopic insertion of PGA may be an extremely effective treatment for post-pancreatitis gastrointestinal fistula.

Endoscopy_UCTN_Code_TTT_1AQ_2AG

Competing interests: None

of Internal Medicine, Showa University

Fujigaoka Hospital, Yokohama, Kanagawa, Japan

\section{References} 2008; 38: $178-183$ could not be identified

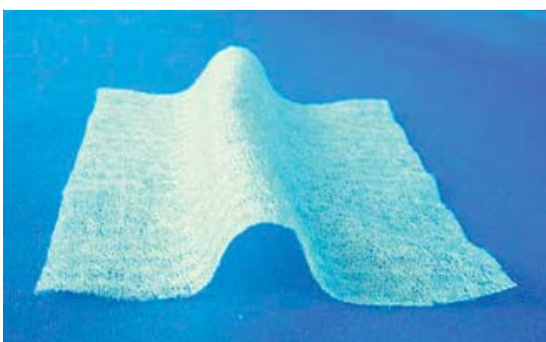

Fig. 5 A polyglycolic acid sheet (Neoveil; Gunze Co., Tokyo, Japan).

Bibliography

DOI http://dx.doi.org/

10.1055/s-0034-1392606

Endoscopy 2015; 47: E424-E425

(c) Georg Thieme Verlag KG

Stuttgart · New York

ISSN 0013-726X

1 Suzuki A, Suzuki S, Sakaguchi T et al. Colonic fistula associated with severe acute pancreatitis: report of two cases. Surg Today

2 Takimoto K, Toyonaga T, Matsuyama K. Endoscopic tissue shielding to prevent delayed perforation associated with endoscopic submucosal dissection for duodenal neoplasms. Endoscopy 2012; 44 (Suppl. 02): E414-415

3 Tsujii Y, Kato M, Shinzaki S et al. Polyglycolic acid sheets for repair of refractory esophageal fistula. Endoscopy 2015; 47 (Suppl. 01): E39-40 